\begin{tabular}{|c|c|}
\hline \multirow{3}{*}{ 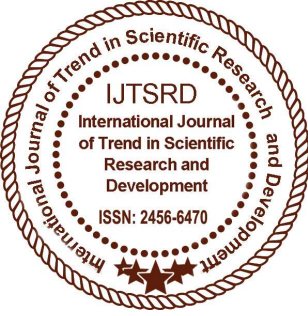 } & $\begin{array}{l}\text { International Journal of Trend in Scientific } \\
\text { Research and Development (IJTSRD) }\end{array}$ \\
\hline & International Open Access Journal \\
\hline & ISSN No: 2456 - 6470 | www.ijtsrd.com | Volume - 2 | Issue - 4 \\
\hline
\end{tabular}

\title{
A Review on: Detecting a specific aspect category for sentiment analysis using association rule mining scheme
}

\author{
Miss. Gayatri D. Khot, Mr. H. A. Tirmare \\ Computer Science and Technology, \\ Department of Technology, Shivaji University Kolhapur, India
}

\begin{abstract}
Now a days online consumer review is most powerful tool for decision making. This term serve as electronic word of mouth (EWM) which become an increasingly popular. Millions of people are now buy products and services via online. Web services are provided this feature to users openly. The web can provides an extensive source of consumer reviews. The user can read all the reviews and evaluate fair view about product or service. This can applicable only for limited number of reviews presented on web. The web contain more than hundreds of reviews then problem arrived and time consuming also. A text processing framework is desirable which summarize all the reviews. This framework would be find out general aspect category addressed in all review sentences. The method presented in this framework which applies association rule mining on co-occurrence frequency data to find out these aspect categories. From this result, generate polarity score for each aspect category. This polarity score helps to evaluate fair decision making for customer as well as company.
\end{abstract}

Keywords: consumer reviews, aspect category, cooccurrence frequency data, polarity score.

\section{Introduction}

Online review facility is now available on every web services which helps to obtain a fair evaluation of product or service. In traditional approach, word of mouth (WoM) technique is used for customer decision making. Word of mouth is an oldest method in which users can share their information by oral communication. Family and friend are important factor for advice and recommendations before any important purchase-decisions can be done. These recommendations can have short term influences as well long term influence on consumer decisionmaking. This WoM technique is greatly expanded. The people who wishes to share their experiences, they can now do so electronically. Social media, like Twitter and IFacebook allow for easy ways to exchange statements about products, services, and brands. The term for this expanded form of WoM is electronic WoM (EWoM).

The outcome that can be gotten from item and services on web is gainful to clients. With the help of this information The customer can decides to buy a product or service as well as this is also beneficial to companies. So that companies knowing what has been posted on the Web can help companies to improve their products or services. If web contains limited number of reviews then system can easily summarize all reviews. In case of large amount of information presented in web reviews, a framework for the automated summarization of reviews is desirable. An important task for such a framework would be to recognize the topics which is people write about. This reorganization is important task for summarization. For example, take a dataset from restaurant review set, the general aspect categories like "food" ,"staff", "atmosphere", "facilities" etc. One of given review sentences is "Food taste is fantastic but staff did not listen properly!" or "Food is not tasty but surrounding environment is very good for family". From this two 
sentences, system classifies reviews to specific aspect category.ie "Food taste is fantastic but staff did not listen properly!" $\rightarrow$ (food) and "Food is not tasty but surrounding environment is very good for family" $\rightarrow$ (atmosphere).From this all specific aspect category classification, system finding out the polarity score to evaluate fair purchase decision making.

\section{LITERATURE REVIEW}

Kim Schouten, Onne van der Weijde, Flavius Frasincar, and Rommert Dekker [1] has proposed 'Supervised and Unsupervised Aspect Category Detection for Sentiment Analysis with Co-Occurrence Data' which focus on sentiment analysis on online customer reviews. The two methods are used ie first one is unsupervised method and second one is supervised method. An unsupervised method that applies association rule mining on co-occurrence frequency data. An Unsupervised method outforms the F1-score of $67 \%$. A supervised method outforms the F1-score of $84 \%$.

Deepa Ananda, Deepan Naorem [2] has proposed Aspect based Sentiment Analysis (ABSA) which focus on interest of users by mining the reviews. The contribution of this paper having two solutions: First is classification scheme with two class without the need for labeled data for plots and reviews is proposed.

Secondly they propose a method to detect aspects and the corresponding opinions using a set of hand crafted rules and aspect clue words.

Walaa Medhat, Ahmed Hassan, Hoda Korashy [3] has proposed 'Sentiment analysis algorithms and applications:A survey' which focus on text mining field. The main target of this survey is to give nearly full image of system analysis techniques and the related fields.

Aitor Garc_a-Pablos, Montse Cuadros, German Rigau[4] has proposed an almost unsupervised system based on topic modelling, that combined with some other unsupervised methods and a minimal configuration, performs aspects category classification.

Xinjie Zhou, Xiaojun Wan and Jianguo Xiao [5] has proposed framework of Representation Learning for Aspect Category Detection in Online Reviews which focus to automatically learn useful features for aspect category detection.
Doaa Mohey El-Din Mohamed Hussein [6] has proposed an article 'A survey on sentiment analysis challenges'. This writer generated sentiment content can be about books, people, hotels, products, research, events, etc.

Md Shad Akhtar, Asif Ekbal, and Pushpak Bhattacharyya[7] has asses the challenges and provide a benchmark setup for aspect category detection and sentiment classification for Hindi.

\section{Alessia D'Andrea,Fernando Ferri,Patrizia}

Grifoni,Tiziana Guzzo[8] gives an overview of the different sentiment classification techniques and tools used for sentiment analysis. It provides a classification of approaches and advantages/limitations and tools.

Samuel Brody, Noemie Elhadad [9] has proposed 'An Unsupervised Aspect-Sentiment Model for Online Reviews' which presents present an unsupervised system for extracting aspects and determining sentiment in review text.

Chetashri Bhadanea,Hardi Dalalb, Heenal Doshic has proposed 'Sentiment analysis: Measuring opinions' which focus on focuses on the various methods used for classifying a given piece of natural language text according to the opinions expressed in it

Emma Haddi, Xiaohui Liu, Yong Shi [11] has proposed 'The Role of Text Pre-processing in Sentiment Analysis' in which sentiment analysis used for text pre-processing and report on experimental results that demonstrate with appropriate feature selection and representation, sentiment analysis accuracies.

Thellaamudhan C, Suresh R, Raghavi P [12] has proposed 'A Comprehensive Survey on Aspect Based Sentiment Analysis' which explains the concept of sentiment analysis which is a growing field in natural language processing to analyze and determine the polarity of given text or data in sentence level or document level.

S Kiritchenko, $X$ Zhu, C Cherry, S Mohammad [13] has given an Abstract Reviews of customers towards various aspects of a product or service. Some of these aspects can be grouped into coarser aspect categories. SemEval-2014 had a shared task on aspect-level sentiment analysis, with over 30 teams participated. 
Jyoti S Deshmukh, Amiya Kumar Tripathy [14] has proposed 'Text Classification using Semisupervised Approach for Multi Domain' which focus on need of domain adaptation algorithms arises to diminish domain's reliance and tagging costs.

Qing Sun, Jianwei Niu, Zhong Yao, Dongmin Qiu has proposed 'Research on Semantic Orientation Classification of Chinese Online Product Reviews Based on Multi-aspect Sentiment Analysis' which apply the Latent Dirichlet Allocation model.

Mondher Bouazizi, Tomoaki Ohtsuki [16] has proposed 'A Pattern-Based Approach for Multi-Class Sentiment Analysis in Twitter' which focus on approach that, in addition to the aforementioned tasks of binary and ternary classification, goes deeper in the classification of texts collected from Twitter and classifies these texts into multiple sentiment classes.

\section{Nipuna Upeka Pannala, Chamira}

\section{Priyamanthi Nawarathna , J.T.K.Jayakody,} Lakmal Rupasinghe, Kesavan Krishnadeva [17] has focus on approach that mainly consist of the way of exploring the sentiment analysis based on the trained data set to provide the positive, negative and neutral reviews for different products in the marketing world.

\section{PROBLEM STATEMENT}

In traditional system, work is done with the help of word of mouth technique. An advice and recommendations are given by orally. However, these works were not created in a perspective for large dataset. Our aim to design a system which applies over large amount of reviews which transformed into specific aspect category. The result is in polarity score which gives an efficiency of product or service.

\section{TOPIC INITIATIVES}

The conventional method is Word of mouth in which users can share their information by oral communication. This method is applicable for limited users i.e. users can communicate with family members or friends or society members and they can share their experience. So this is one kind drawback of oldest method that is limited area.

This drawback is overcome by upgraded method. A portion of the fundamental destinations are:

To study and implement methods to find set of aspect categories from the co-occurrence data.
$>$ Implement frequent word list.

$>$ Analyze extracting opinioned summary.

$>$ To generate activation value for each aspect category.

$>$ To generate polarity score and predict the aspect category.

\section{OUTLINE OF PROPOSED WORK}

The method is proposed that are able to find aspect categories based on co-occurrence frequencies. The system take the input as customer's reviews. The natural language processing is done on this reviews to remove streaming and stopping words. Applying the association rule mining to find out the appropriate aspect category for particular review. The proposed system gives a framework to detecting the specific aspect category for general review sentences and then applies association rule mining scheme which result in review report and result measurement i.e. polarity score.

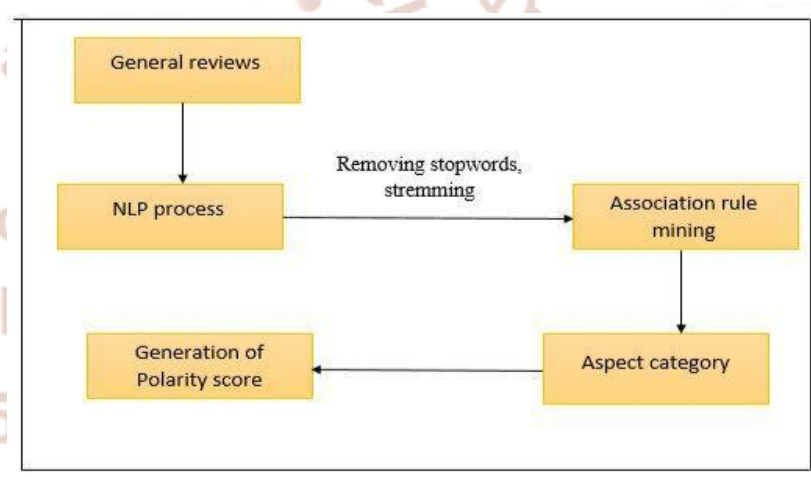

\section{System Architecture}

\section{CONCLUSION}

Customer's reviews are opinion about products which buy online. With the help of this reviews, customer's decides to buy the products online or not. Traditional technique was provide the frame for limited number of reviews presented on web. But most of webs contains more than thousands of reviews. This processing may take longer time. The proposed system process the NLP on reviews which remover stopping and streaming words from reviews. The framework finds general aspect category for each reviews. The system calculates positive and negative score for each sentence. The result show the graph for all aspect category based on positive and negative score.For this score,system calculates polarity score which is used for customer's decision making process. 


\section{REFERENCES}

1) Kim Schouten, Onne van der Weijde, Flavius Frasincar, and Rommert Dekker,'Supervised and Unsupervised Aspect Category Detection for Sentiment Analysis With Co-Occurrence Data" IEEE TRANSACTIONS ON CYBERNETICS, Volume: PP, Issue: 99, April 2017.

2) Deepa Ananda, Deepan Naorem," Semisupervised Aspect Based Sentiment Analysis for Movies using Review Filtering” 7th International conference on Intelligent Human Computer Interaction, IHCI 2015.

3) Walaa Medhat, Ahmed Hassan, Hoda Korashy," Sentiment analysis algorithms and applications: A survey", Ain Shams Engineering Journal (2014) 5, 1093-1113.

4) Aitor Garc_a-Pablos, MontseCuadros, German Rigau," W2VLDA: Almost Unsupervised System for Aspect Based Sentiment Analysis", July 19, 2017.

5) Xinjie Zhou, Xiaojun Wan and Jianguo Xiao," Representation Learning for Aspect Category Detection in Online Reviews", Twenty-Ninth AAAI Conference on Artificial Intelligence.

6) Doaa Mohey El-Din Mohamed Hussein," A survey on sentiment analysis challenges", Journal of King Saud University - Engineering Sciences (2016),

7) Md Shad Akhtar, Asif Ekbal, and Pushpak Bhattacharyya," Aspect Based Sentiment Analysis: Category Detection and Sentiment Classification for Hindi", Indian Institute of Technology Patna India-801103.

8) Alessia D’Andrea,Fernando Ferri,Patrizia Grifoni,Tiziana Guzzo," Approaches, Tools and Applications for Sentiment Analysis Implementation", International Journal of Computer Applications (0975 - 8887) Volume 125 - No.3, September 2015.

9) Samuel Brody, Noemie Elhadad," An Unsupervised Aspect-Sentiment Model for Online Reviews", The 2010 Annual Conference of the North American Chapter of the ACL, pages 804812 .

10) Chetashri Bhadanea,Hardi Dalalb, Heenal Doshic," Sentiment analysis: Measuring opinions", International Conference on Advanced
Computing Technologies and Applications (ICACTA-2015).

11) Emma Haddi, Xiaohui Liu, Yong Shi," The Role of Text Pre-processing in Sentiment Analysis", Information Technology and Quantitative Management (ITQM2013).

12) Thellaamudhan C, Suresh R, Raghavi P," A Comprehensive Survey on Aspect Based Sentiment Analysis", International Journal of Advanced Research in Computer Science and Software Engineering, Volume 6, Issue 4, April 2016.

13) S. Kiritchenko, X. Zhu, C. Cherry, and S. M. Mohammad, "NRCCananda-2014: Detecting aspects and sentiment in customer reviews,"in Proc. 8th Int. Workshop Semantic Eval. (SemEval), Dublin, Ireland, 2014, pp. 437442.

14) Jyoti S Deshmukh, Amiya Kumar Tripathy," Text Classification using Semi-supervised Approach for Multi Domain”, 2017 International Conference on Nascent Technologies in the Engineering Field (ICNTE-2017).

15) Qing Sun , Jianwei Niu, Zhong Yao, Dongmin Qiu," Research on Semantic Orientation Classification of Chinese Online Product Reviews Based on Multi-Aspect Sentiment Analysis", Big Data Computing Applications and Technologies (BDCAT), 2016 IEEE/ACM 3rd International Conference.

16) Mondher Bouazizi, Tomoaki - Ohtsuki," A Pattern-Based Approach for Multi-Class Sentiment Analysis in Twitter", IEEE Access (Volume: PP, Issue: 99 ).

17) Nipuna Upeka Pannala, Chamira Priyamanthi Nawarathna , J.T.K.Jayakody, Lakmal Rupasinghe, Kesavan Krishnadeva," Supervised Learning Based Approach to Aspect Based Sentiment Analysis", 2016 IEEE International Conference on Computer and Information Technology.

18) Y. Tang, Y.-Q. Zhang, N. V. Chawla, and S. Krasser, "SVMs modeling for highly imbalanced classification," IEEE Trans. Syst., Man, Cybern. B, Cybern., vol. 39, no. 1, pp. 281-288, Feb. 2009. 
19) T. Brychcin, M. Konkol, and J. Steinberger, "UWB: Machine learning approach to aspectbased sentiment analysis," in Proc. 8th Int. Workshop Semantic Eval. (SemEval), Dublin, Ireland, 2014, pp. 817-822.

20) A. Garcia-Pablos, M. Cuadros, S. Gaines, and G. Rigau, "V3: Unsupervised generation of domain aspect terms for aspect based sentiment analysis," in Proc. 8th Int. Workshop Semantic Eval. (SemEval), Dublin, Ireland, 2014, pp. 833-837.

21) R. Feldman, "Techniques and applications for sentiment analysis,"Commun. ACM, vol. 56, no. 4, pp. 82-89, 2013.

22) S. Sen and D. Lerman, "Why are you telling me this? An examination into negative consumer reviews on the Web," J. Interact. Marketing, vol. 21, no. 4, pp. 76-94, 2007.

23) D. Smith,S.Menon, and K.Sivakumar, "Online peer and editorial recommendations, trust, and choice in virtual markets," J. Interact. Marketing, vol. 19, no. 3, pp. 15-37, 2005.

24) A. Katifori, C. Vassilakis, and A. Dix, "Ontologies and the brain: Using spreading activation through ontologies to support personal interaction," Cognitive Syst. Res., vol. 11 , no. 1 , pp. 25-41, 2010.

25) SemEval-2014 dataset which contain both training sets and test SSN sets [Online].Available:https://github.com/pedrobalage /SemevalAspectBasedSentimentAnalysis/tr ee/master/semeval_data

26) Linguist. Syst. Demonstrations, 2014, pp. 5560.[Online].Available: http://www.aclweb.org/anthology/P/P14/P14$\underline{5010}$.

27) X. Zheng, Z. Lin, X. Wang, K.-J. Lin, and M. Song, "Incorporating appraisal expression patterns into topic modeling for aspect and sentiment word identification," Knowl. Based Syst., vol. 61, no. 1, pp. 29-47,2014.

28) G. Castellucci, S. Filice, D. Croce, and R. Basili, "UNITOR: Aspect based sentiment analysis with structured learning," in Proc. $8^{\text {th }}$ Int. Workshop Semantic Eval. (SemEval), Dublin, Ireland, 2014,pp. 761-767.

29) K. Schouten and F. Frasincar, "Survey on aspectlevel sentiment analysis," IEEE Trans. Knowl. Data Eng., vol. 28, no. 3, pp. 813-830,Mar. 2016.

30) Wikipedia. needs". "Fundamental human https://en.Wikipedia.org/wiki/Fundame ntal_human_needs [Apr. 29, 2016].

31) G.Angulakshmi, .R.ManickaChezian, "An Analysis on Opinion Mining: Techniques and Tools." IT International Journal of Advanced Research in Computer and Communication Engineering, Vol. 3, Issue 7,July 2014.

32) B. Bickart and R. M. Schindler, "Internet Forums as Influential Sources of Consumer Information," Journal of Interactive Marketing, vol. 15, no. 3, pp. 31-40, 2001. 